\title{
Small- bowel mucosal changes and antibody responses after low- and moderate-dose gluten challenge in celiac disease
}

\author{
Marja-Leena Lähdeaho ${ }^{1}$, Markku Mäki ${ }^{1}$, Kaija Laurila ${ }^{1}$, Heini Huhtala ${ }^{2}$ and Katri Kaukinen ${ }^{3^{*}}$
}

\begin{abstract}
Background: Due to the restrictive nature of a gluten-free diet, celiac patients are looking for alternative therapies. While drug-development programs include gluten challenges, knowledge regarding the duration of gluten challenge and gluten dosage is insufficient.

We challenged adult celiac patients with gluten with a view to assessing the amount needed to cause some smallbowel mucosal deterioration.

Methods: Twenty-five celiac disease adults were challenged with low (1-3 g) or moderate (3-5g) doses of gluten daily for 12 weeks. Symptoms, small-bowel morphology, densities of CD3+ intraepithelial lymphocytes (IELs) and celiac serology were determined.

Results: Both moderate and low amounts of gluten induced small-bowel morphological damage in $67 \%$ of celiac patients. Moderate gluten doses also triggered mucosal inflammation and more gastrointestinal symptoms leading to premature withdrawals in seven cases. In $22 \%$ of those who developed significant small- intestinal damage, symptoms remained absent. Celiac antibodies seroconverted in $43 \%$ of the patients.

Conclusions: Low amounts of gluten can also cause significant mucosal deterioration in the majority of the patients. As there are always some celiac disease patients who will not respond within these conditions, sample sizes must be sufficiently large to attain to statistical power in analysis.
\end{abstract}

\section{Background}

Celiac disease is an autoimmune-like systemic disorder in genetically susceptible individuals, perpetuated by daily ingested gluten cereals wheat, rye and barley and with manifestations in the small- intestinal mucosa and in organs outside the gut. The gold standard for celiac disease diagnosis is the finding of gluten-induced smallintestinal mucosal injury $[1,2]$. The mucosa will heal upon introduction of a gluten-free diet and the mucosal damage will reappear if gluten is reintroduced [2]. Extensive time-course studies have provided evidence that during gluten challenge an inflammatory process with a dose-dependent accumulation of intraepithelial lymphocytes (IELs) is followed by mucosal villous atrophy with

\footnotetext{
* Correspondence: katri.kaukinen@uta.fi

${ }^{3}$ Department of Gastroenterology and Alimentary Tract Surgery, Tampere University Hospital and School of Medicine, University of Tampere, Tampere, Finland

Full list of author information is available at the end of the article
}

crypt hyperplasia [3]. In untreated celiac disease the degree of malabsorption is determined by the length of the functionally impaired bowel and the presence of symptoms is noway related to the histological features of a proximal biopsy [3-5]. More recently, it was concluded that only patients with extensive and severe enteropathy will give evidence of steatorrhea and increased intestinal permeability[6]. Biopsy has thus remained the golden standard in measuring gluten-induced ill health.

Previously a gluten challenge was part of the routine diagnostic procedure in children and a characteristic mucosal lesion reappeared in most patients within two years[2,7]. In gluten challenge studies, the amount of gluten in normal food has been estimated to be as high as $10-20$ g per day[8-10]. A reduced daily gluten dose (2.5-5 g per day) during a gluten-free diet has still enabled mucosal healing in half of the patients [11]. Also some adults challenged with 2.5-5 g of gluten daily for three to 14 months have shown no significant

\section{Ciomed Central}


changes in mucosal morphology [12]. These findings are in marked conflict with those from a recent challenge study where it was shown that even a minimal dose of $50 \mathrm{mg}$ gluten daily induced a significant mucosal deterioration [13]. Altogether, the patient groups in these studies have been rather small and no unequivocal conclusions can be drawn.

A gluten-free diet is socially troublesome and expensive and research has shown compliance to the diet often to be poor [14]. Gluten contamination in a gluten-free diet is also difficult to avoid $[15,16]$. For these reasons many celiac patients have expressed a desire for other alternative or complementary treatments, less burdensome than a strict life-long gluten-free diet $[17,18]$. Any drug under evaluation as clinically effective and trusted by celiac disease patients should significantly reduce or prevent gluten-induced mucosal injury. However, data are insufficient as to the amount of gluten to be used in a challenge study and for how long.

In this pilot study we challenged adult celiac disease patients with gluten aiming to gain a clearer conception of the gluten amount needed to cause some mucosal deterioration but without inducing excessive ill health with a resultant dropout of trial subjects. We challenged treated celiac disease patients with low to moderate amounts of gluten for up to 12 weeks to establish whether a morphometrically measurable mucosal deterioration could be detected. We especially measured separately mucosal morphological change as villous height crypt depth ratio $(\mathrm{Vh} /$ $\mathrm{CrD}$ ) and the inflammatory aspect in terms of density of mucosal IELs, both known to be sensitive continuous functional parameters of gluten ingestion in celiac disease. Further, we sought to establish whether the gluten-dependent serum antibody markers show seroconversions.

\section{Methods}

\section{Patients and study design}

Altogether 25 adult volunteers with previously diagnosed celiac disease were recruited for the gluten challenge study. Inclusion criteria comprised biopsy-proven celiac disease in patients aged $18-75$ years adhering to a strict gluten-free diet for at least two years and being in clinical remission as judged by an interview, clinical examination and on-site rapid celiac autoantibody testing. Patients with a history of regular dietary transgressions, and those suffering from a condition carrying a risk at endoscopy, or using immunosuppressive medication were excluded.

At the outset the symptoms or signs leading to the diagnosis of celiac disease, the duration of the gluten-free diet, family history of celiac disease and associated conditions were recorded. Upper gastrointestinal endoscopy with duodenal biopsies, laboratory analysis and clinical evaluation including gastrointestinal symptoms, were carried out at baseline and after the challenge. The complete challenge lasted 12 weeks ( $84 \pm 14$ days). Furthermore, at 4 weeks ( $28 \pm 7$ days) and 8 weeks ( $56 \pm 14$ days) the clinical and serological assessments were repeated. The study patients were divided into two groups, those consuming a moderate (3-5 g) amount of gluten and those consuming a low (1-3 g) amount of gluten daily. Those who prematurely withdrew from the study were encouraged to undergo follow-up examinations immediately after the challenge was discontinued. After the challenge all patients continued their normal gluten-free diet.

\section{Gluten product and dietary assessment}

Each patient consumed 1.25-5 g of gluten (as a biscuit) daily during the challenge while otherwise continuing on a strict gluten-free diet. A verified amount of industrial gluten (Meelunie, Amsterdam, the Netherlands) was added to the baking mixture of the biscuits, and the mean daily gluten consumption of each patient was calculated. Consumption of the gluten product, changes in dietary habits and possible dietary transgressions were inquired at each visit and by regular telephone contacts. At baseline and at the end of the study a dietitian made a detailed dietary analysis by means of interview and a 4-day record of food intake.

\section{Small-bowel mucosal morphology and inflammation}

Up to seven small-bowel biopsy specimens were taken from the distal part of the duodenum upon upper gastrointestinal endoscopy; the specimens were evaluated by the same investigator without prior knowledge of the history or findings. At least three biopsies were processed and stained with hematoxylin-eosin and studied under light microscopy. Poorly oriented sections were discarded; when necessary, the samples were dissected repeatedly until they were of good quality. Morphometric analysis measuring $\mathrm{Vh} / \mathrm{CrD}$ was made in well-oriented biopsy samples as previously described [19], and a ratio $<2.0$ was regarded as compatible with villous atrophy and crypt hyperplasia and indicative of active celiac disease. During the gluten-challenge, a decrease in $\mathrm{Vh} / \mathrm{CrD}$ of 0.5 or more was considered significant and equivalent to clinical glutensensitivity [20].

The remaining biopsies were snap-frozen for immunohistochemical stainings. The specimens were freshly embedded in optimal temperature compound (OCT, Tissue-Tec, Miles Inc, Elkhart, IN, USA), snap-frozen in liquid nitrogen and stored at $-70^{\circ} \mathrm{C}$. Immunohistochemical studies were carried out on 5 - $\mu$ m-thick frozen sections. CD3+ IELs were stained with monoclonal antibody Leu-4 (Becton Dickinson, San Jose, CA, USA). IELs were counted with a x100 flat-field light microscope objective in randomly selected surface epithelium and the density of IELs expressed as cells/millimetre of epithelium as previously described, the reference values being set at 
37 cells/mm for CD3+ IELs [21]. After the gluten challenge an over $30 \%$ increase in IEL counts was considered significant and equivalent to clinical glutensensitivity [20]. In our laboratory the correlation coefficients for intraobserver variation for CD3+ IELs has been 0.95 and that for interobserver variation 0.92 .

\section{Celiac serology}

Serum IgA class transglutaminase 2 (TG2)-antibodies were investigated by an enzyme-linked immunosorbent assay (ELISA) according to manufacturer's instructions (QUANTA Lite h-tTGIgA, INOVA, Diagnostics, Inc, CA, USA). The cut-off value for a positive antibody level was 20 IU. Serum IgA class endomysial antibodies (EMA) were determined by an indirect immunofluorescence method using human umbilical cord as substrate; a dilution of 1:5 or more was considered positive [22]. Positive sera were further diluted: 1:50, 1:100, 1:200, 1:500, 1:1000, 1:2000, 1:4000. We also determined a combination of serum IgA and IgG class deaminated gliadin peptide (DGP) antibodies (QUANTA Lite Celiac DPG Screen, INOVA Diagnostics) by ELISA, and values $\geq 20$ IU were considered positive. None of the study subjects suffered from selective IgA deficiency. The change in antibody levels was considered clinically significant when antibodies seroconverted from negative to positive or when positive antibody levels increased and at least doubled during the gluten challenge.

Small-bowel mucosal TG2-targeted autoantibody deposits The small bowel mucosal TG2- specific autoantibody deposits were studied by direct immunofluorescence methods in frozen biopsy sections [20,23-25]. From each patient altogether six unfixed, 5 - $\mu \mathrm{m}$-thick sections from frozen small-bowel specimens were processed, three for investigation of IgA deposits and three for double-color labelling for both IgA and TG2. IgA was detected by direct immunofluorescence using fluorescein isothiocyanate-labelled rabbit antibody against human IgA (Dako AS, Glostrup, Denmark) as previously described [23]. In celiac disease a clear subepithelial IgA deposition was found below the basement membrane along the villous and crypt epithelium and around mucosal vessels, in contrast to that observed in non-celiac samples, where only endogenous IgA is found inside plasma cells and epithelial cells. Sections were further double-stained for human IgA (green, as above) and for TG2 (red, monoclonal mouse antibody CUB7402, NeoMArers, Fremont, CA, USA, followed by rhodamine-conjugated anti-mouse immunoglobulin antibodies, Dako) to confirm that celiac-type IgA deposits co-localized with TG2. The occurrence of IgA deposits was graded semi-quantitatively according to their intensity along the basement membrane in the villous-crypt area as follows: negative $(0)$, weak $(+)$, moderate $(++)$ and strong positive $(+++)$. In cases where the intensity of the staining was patchy, it was graded from different areas and the mean value was given. All evaluations were carried out in blinded fashion without knowledge of disease history or laboratory findings. The correlation coefficients for both intraobserver and interobserver variations for the detection of presence or absence of TG2-specific IgA deposits have been 0.98 in our laboratory. An increase in the intensity of IgAdeposit staining after gluten challenge was considered indicative of clinical gluten-sensitivity.

\section{Gastrointestinal symptoms}

Clinical symptoms appearing during the gluten challenge were semiquantatively classified into four categories- no symptoms, slight, moderate or severe symptoms- by means of interview and recorded at each visit.

\section{Statistics}

Quantitative data were expressed as medians and ranges or means and $95 \%$ confidence intervals (CI). Statistical differences were evaluated using Mann-Whitney test and Wilcoxon's test or unpaired and paired t-test as appropriate, and all testing was two-sided. Fisher's exact test was used in cross-tabulations. The Pearson coefficient was used for correlation studies. P-values $<0.05$ were considered statistically significant. All statistical testing was performed using SPSS version 16.0 (SPSS Inc., Chicago, IL, USA)

\section{Ethical considerations}

The study protocol was approved by the Ethics Committee of Tampere University Hospital. All subjects gave written informed consent.

\section{Results}

Altogether 25 adult celiac disease patients fulfilling the inclusion criteria were enrolled for the study (Table 1). At the time of the diagnosis, the majority $(88 \%)$ had suffered from abdominal symptoms. When volunteering for this study, the patients had been a median of 11 years on a strict gluten-free diet, and all were in clinical remission.

At baseline in the current study, all patients except one had normal $\mathrm{Vh} / \mathrm{CrD}$ ratios (median 2.9, range 1.3-4.2) on small-intestinal biopsy (Table 2). The mean density of CD3+ IELs was 63 cells/mm (range 23-146). Altogether six patients had borderline positive celiac disease antibody levels (three had positive TG2 antibodies, two EMA and one DGP antibodies); in the rest all three antibodies were negative. When TG2-targeted autoantibodies were assessed at small-bowel mucosal level, moderate IgAdeposits were seen in two and faint in four patients. In 
Table 1 Demographic data on the 25 celiac disease patients

\begin{tabular}{ll}
\hline Variable & \\
\hline Female; $\mathrm{n}$ & $21(81 \%)$ \\
Age; median (range), years & $49(21-68)$ \\
Reason for suspicion of celiac disease at diagnosis & \\
$\quad$ Abdominal symptoms & $23(88 \%)$ \\
$\quad$ Anemia or malabsorption & $16(62 \%)$ \\
$\quad$ Extraintestinal manifestations* & $9(35 \%)$ \\
$\quad$ Screening in risk groups of celiac diseaset & $1(4 \%)$ \\
Duration of gluten free diet; median (range), years & $11(2-34)$ \\
Family history of celiac disease, $\mathrm{n}$ & $8(31 \%)$ \\
\hline
\end{tabular}

*tooth enamel hypoplasia, hypothyreosis, growth delay

† family history of celiac disease the remaining 19 cases, mucosal TG2-autoantibody depositions were negative at the beginning of the challenge study.

Altogether four celiac disease patients belonging to the moderate gluten dose group discontinued the study due to abdominal symptoms shortly after the challenge was initiated (after three, eight, ten and 23 days), and they were excluded from the final analyses. Celiac serology remained negative in all. The first two drop-outs with extremely brief gluten challenges underwent the followup endoscopy, and no small- bowel mucosal deterioration was evident.

The remaining 21 patients comprised the final study population (Tables 2 and 3), for whom the gluten challenge lasted a median of 84 days (range 29-103 days)

Table 2 Effect of moderate (3-5g) and low (1-3g) amounts of gluten on small-bowel mucosal villous height crypt depth ratio (Vh/CrD), densities of CD3+ intraepithelial lymphocytes (IELs) and gastrointestinal symptoms in treated celiac disease patients

\begin{tabular}{|c|c|c|c|c|c|c|c|c|}
\hline \multirow[t]{3}{*}{ No } & \multirow[t]{3}{*}{ Mean daily gluten intake (g) } & \multirow[t]{3}{*}{ Duration of gluten challenge (days) } & \multicolumn{4}{|c|}{ Small-bowel biopsy } & \multicolumn{2}{|c|}{ Symptoms } \\
\hline & & & \multicolumn{2}{|c|}{$\mathrm{Vh} / \mathrm{CrD}^{*}$} & \multicolumn{2}{|c|}{ CD3+ IELs† } & & \\
\hline & & & 1 & II & 1 & II & 1 & II \\
\hline \multicolumn{9}{|c|}{ Moderate amounts of gluten daily } \\
\hline 1 & 5.0 & 29 & 2.8 & 0.8 & 33 & 88 & 0 & ++ \\
\hline 2 & 4.9 & 84 & 3.5 & 3.4 & 43 & 84 & 0 & 0 \\
\hline 3 & 4.9 & 38 & 2.9 & 1.3 & 64 & 128 & 0 & ++ \\
\hline 4 & 4.7 & 45 & 2.7 & 0.2 & 70 & 113 & 0 & + \\
\hline 5 & 4.1 & 61 & 3.0 & 3.5 & 41 & 56 & 0 & ++ \\
\hline 6 & 4.0 & 91 & 3.0 & 0.6 & 45 & 97 & 0 & + \\
\hline 7 & 3.6 & 91 & 2.8 & 1.4 & 42 & 125 & 0 & 0 \\
\hline 8 & 3.6 & 84 & 3.0 & 2.6 & 38 & 47 & 0 & + \\
\hline 9 & 3.4 & 88 & 3.8 & 2.3 & 69 & 83 & 0 & + \\
\hline 10 & 3.3 & 86 & 2.7 & 0.6 & 68 & 96 & 0 & + \\
\hline \multicolumn{9}{|c|}{ Low amounts of gluten daily } \\
\hline 11 & 2.8 & 89 & 2.5 & 3.1 & 87 & 52 & 0 & + \\
\hline 12 & 2.7 & 84 & 3.0 & 0.6 & 87 & 117 & 0 & 0 \\
\hline 13 & 2.6 & 81 & 2.9 & 3.1 & 49 & 50 & 0 & 0 \\
\hline 14 & 2.4 & 85 & 2.7 & 1.9 & 146 & 271 & 0 & + \\
\hline 15 & 2.2 & 103 & 4.2 & 1.3 & 23 & 48 & 0 & + \\
\hline 16 & 2.1 & 93 & 1.3 & 0.1 & 130 & 99 & 0 & + \\
\hline 17 & 2.1 & 85 & 3.3 & 2.4 & 100 & 79 & 0 & + \\
\hline 18 & 2.1 & 84 & 2.9 & 1.7 & 75 & 78 & 0 & 0 \\
\hline 19 & 2.1 & 83 & 3.2 & 3.4 & 70 & 123 & 0 & + \\
\hline 20 & 1.4 & 77 & 3.4 & 3.0 & 41 & 87 & 0 & ++ \\
\hline 21 & 1.3 & 78 & 2.5 & 0.8 & 53 & 71 & 0 & 0 \\
\hline \multicolumn{3}{|c|}{ Altogether positive outcome§, n (\%) } & \multicolumn{2}{|c|}{$14(67 \%)$} & \multicolumn{2}{|c|}{$14(67 \%)$} & \multicolumn{2}{|c|}{$15(71 \%)$} \\
\hline
\end{tabular}

Clinically significant changes between baseline (I) and 12 weeks' follow-up examination (II) are marked in bold numbers. Complete challenge lasted 12 weeks ( 84 \pm 14 days).

* Abnormal ratio compatible with active celiac disease $<2.0$

† Reference value for CD3+ IELs 37 cell $/ \mathrm{mm}$

‡ Symptoms graded as $0=$ no symptoms, $+=$ mild symptoms, ++ = moderate symptoms,

$\S$ Positive outcome reported when clinically significant changes appeared ( $>0.5$ decrease in $\mathrm{Vh} / \mathrm{CrD},>30 \%$ increase in IEL densities) or when symptoms worsened. 
Table 3 Effect of moderate (3-5g) and low (1-3g) amounts of gluten on serum transglutaminase 2 (TG2) antibodies and small bowel mucosal TG2-targeted autoantibody deposits in treated celiac disease patients

\begin{tabular}{|c|c|c|c|c|c|c|}
\hline \multirow[t]{2}{*}{ No } & \multirow[t]{2}{*}{ Mean daily gluten intake } & \multirow[t]{2}{*}{ Duration of gluten } & \multicolumn{2}{|c|}{ Serum TG2 antibodies* } & \multicolumn{2}{|c|}{ Mucosal TG2 antibody depositst } \\
\hline & & & 1 & II & $\mathrm{I}$ & II \\
\hline \multicolumn{7}{|c|}{ Moderate amounts of gluten daily } \\
\hline 1 & 5.0 & 29 & 10 & 319 & 0 & +++ \\
\hline 2 & 4.9 & 84 & 9 & 13 & 0 & 0 \\
\hline 3 & 4.9 & 38 & 5 & 10 & 0 & + \\
\hline 4 & 4.7 & 45 & 44 & 218 & + & +++ \\
\hline 5 & 4.1 & 61 & 6 & 5 & 0 & 0 \\
\hline 6 & 4.0 & 91 & 21 & 263 & + & +++ \\
\hline 7 & 3.6 & 91 & 6 & 47 & 0 & ++ \\
\hline 8 & 3.6 & 84 & 8 & 10 & ++ & + \\
\hline 9 & 3.4 & 88 & 13 & 16 & 0 & 0 \\
\hline 10 & 3.3 & 86 & 18 & 229 & + & +++ \\
\hline \multicolumn{7}{|c|}{ Low amounts of gluten daily } \\
\hline 11 & 2.8 & 89 & 6 & 7 & 0 & ++ \\
\hline 12 & 2.7 & 84 & 7 & 111 & 0 & ++ \\
\hline 13 & 2.6 & 81 & 7 & 5 & 0 & 0 \\
\hline 14 & 2.4 & 85 & 12 & 88 & 0 & ++ \\
\hline 15 & 2.2 & 103 & 9 & 9 & 0 & 0 \\
\hline 16 & 2.1 & 93 & 16 & 94 & ++ & ++ \\
\hline 17 & 2.1 & 85 & 9 & 8 & 0 & 0 \\
\hline 18 & 2.1 & 84 & 24 & 21 & + & + \\
\hline 19 & 2.1 & 83 & 8 & 8 & 0 & 0 \\
\hline 20 & 1.4 & 77 & 10 & 13 & 0 & + \\
\hline 21 & 1.3 & 78 & 14 & 101 & 0 & ++ \\
\hline \multicolumn{3}{|c|}{ Altogether positive outcome $\neq, \mathrm{n}(\%)$} & \multicolumn{2}{|c|}{$9(43 \%)$} & \multicolumn{2}{|c|}{$11(52 \%)$} \\
\hline
\end{tabular}

Clinically significant changes between baseline (I) and 12 weeks' follow-up examination (II) are marked in bold letters. Complete challenge lasted 12 weeks ( $84 \pm$ 14 days).

* Values of $\geq 20$ IU considered positive

† Graded as negative (0), weak (+), moderate (++) and strong positive (+++)

₹ Positive outcome reported when serum antibody levels or intensity of mucosal autoantibody deposits increased.

with an average of $3.1 \mathrm{~g}$ daily gluten consumption (range 1.3-5.0 g/day). When the cohort was further divided according to the average daily gluten intake, ten patients belonged to the moderate-dose (3-5 g) and 11 to the low-dose (1-3 g) gluten challenge group (Tables 2 and 3$)$. In the moderate-dose group, three patients withdrew prematurely (after 29, 38 and 61 days) by reason of abdominal symptoms such as diarrhea, vomiting and abdominal pain; one patient discontinued at day 45 for personal reasons. Intended end-point examinations were carried out in all four cases immediately after cessation of the challenge. All celiac patients in the low-dose gluten challenge group completed the study as planned after 12 weeks' challenge. Patients with a moderate gluten dose were thus more prone than those with a low dose to withdraw prematurely from the study $(\mathrm{p}=$ 0.035).

During the gluten challenge, a clinically significant decrease in $\mathrm{Vh} / \mathrm{CrD}$ was found in $14(67 \%)$ out of the 21 celiac patients; seven were in the moderate and seven in the low gluten dose group (Table 2, Figures 1 and 2). The $\mathrm{Vh} / \mathrm{CrD}$ in the moderate-dose group deteriorated from a mean 3.0 (95\%CI $2.8-3.2)$ to 1.7 (95\%CI 1.0-2.4) and in the low-dose group from 2.9 (95\%CI $2.5-3.4)$ to $1.9(95 \%$ CI 1.2-2.6), both changes being statistically significant ( $\mathrm{p}$ $=0.002$ and $\mathrm{p}=0.016$, respectively). The changes in $\mathrm{Vh} /$ $\mathrm{CrD}$ did not correlate with daily gluten intake $(\mathrm{r}=-0.138$, $\mathrm{p}=0.552)$.

In line with the morphological findings, a clinically significant change in the density of CD3+ IELs was seen in altogether $14(67 \%)$ subjects; eight in the moderate-dose and six in the low-dose of (Table 2, Figures 3 and 4). In the moderate gluten dose group the IEL count increased significantly from a mean $49 \mathrm{cell} / \mathrm{mm}(95 \% \mathrm{CI} 38-60)$ to $88 \mathrm{cell} / \mathrm{mm}(95 \% \mathrm{CI} 70-106, \mathrm{p}=0.001)$. In the low-dose group the increase did not reach statistical significance (from a mean $78 \mathrm{cell} / \mathrm{mm}$ [95\%CI 56-100] to $98 \mathrm{cell} / \mathrm{mm}$ [95\%CI 61-135], $\mathrm{p}=0.188)$. All in all, daily gluten intake did not correlate with increased inflammation (gluten intake vs. $\triangle \mathrm{CD} 3+\mathrm{IEL}$ count, $\mathrm{r}=0.251, \mathrm{p}=0.273$ ). 


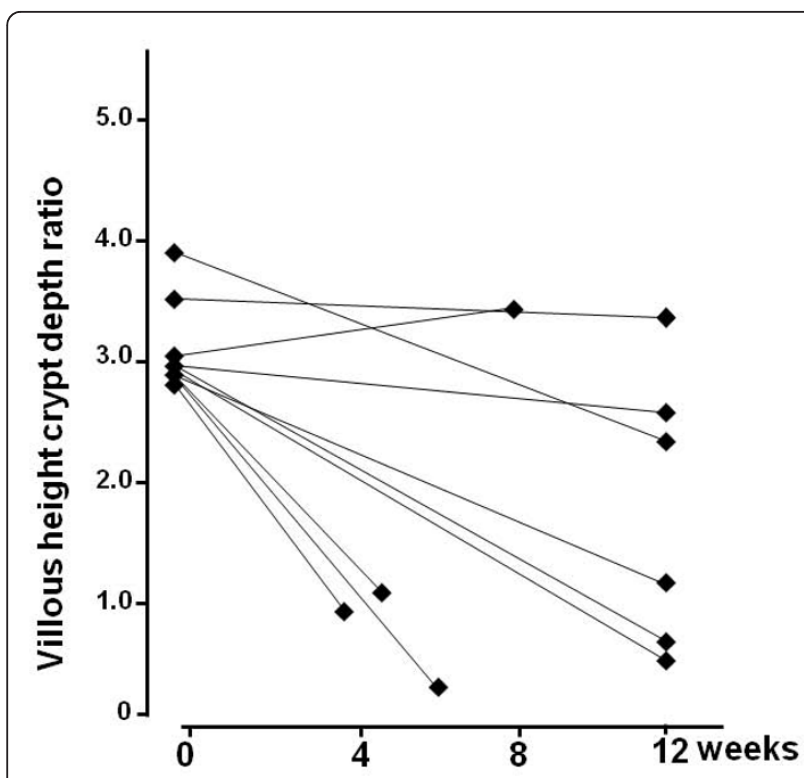

Figure 1 Changes in small bowel mucosal villous height and crypt depth ratios in moderate-dose group at baseline and at completion of the study.

Altogether nine (43\%) celiac disease patients, five in the moderate- and four in the low-dose gluten challenge group, showed significant increases in TG2 antibody titers (Table 3). The subjects consuming moderate doses of gluten evinced clearly higher TG2 antibody titer responses than those consuming low doses; this was already seen after four weeks' challenge (Figure 5). Interestingly, after the highest positive titer during the gluten challenge, the

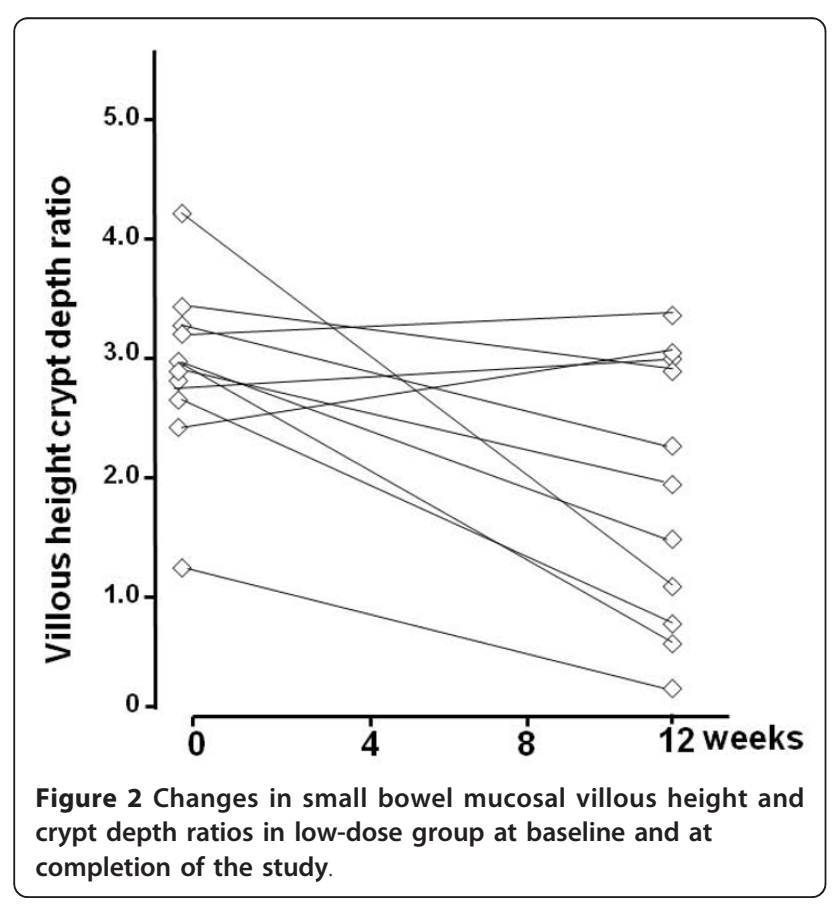

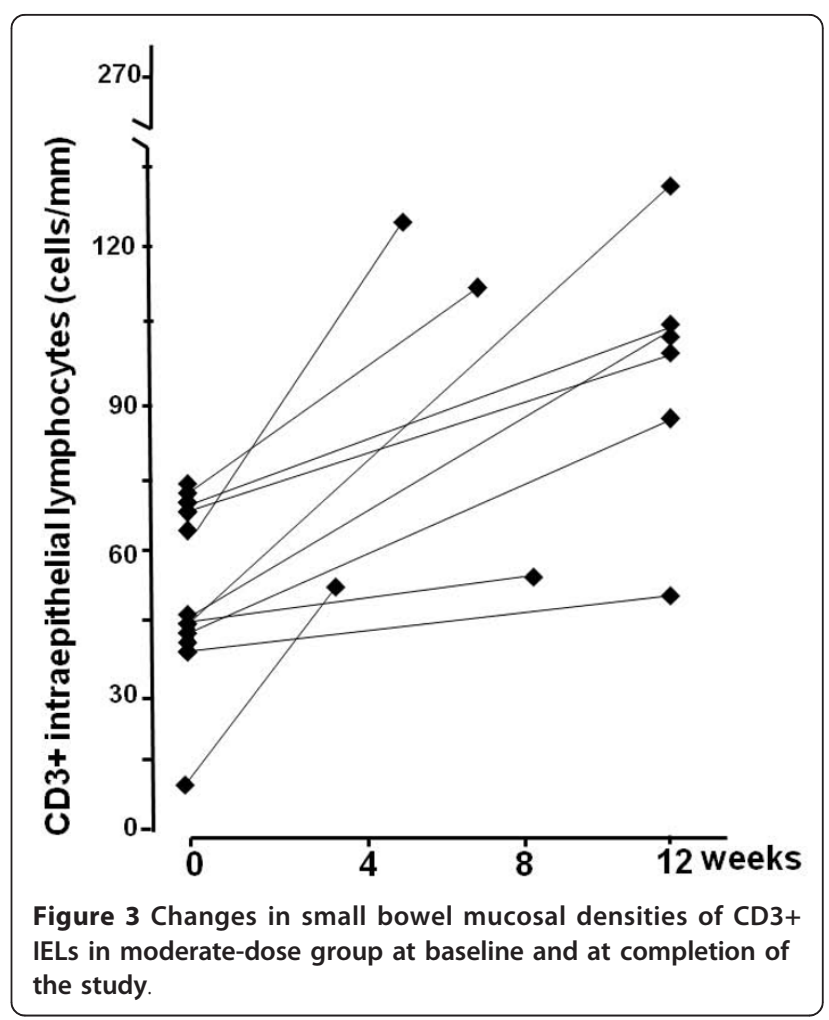

serum TG2 antibody levels tended to decrease even if gluten consumption continued (Figure 5). Serum EMA and DGP antibody titers were in line with TG2 antibody results; furthermore, after the gluten challenge all these

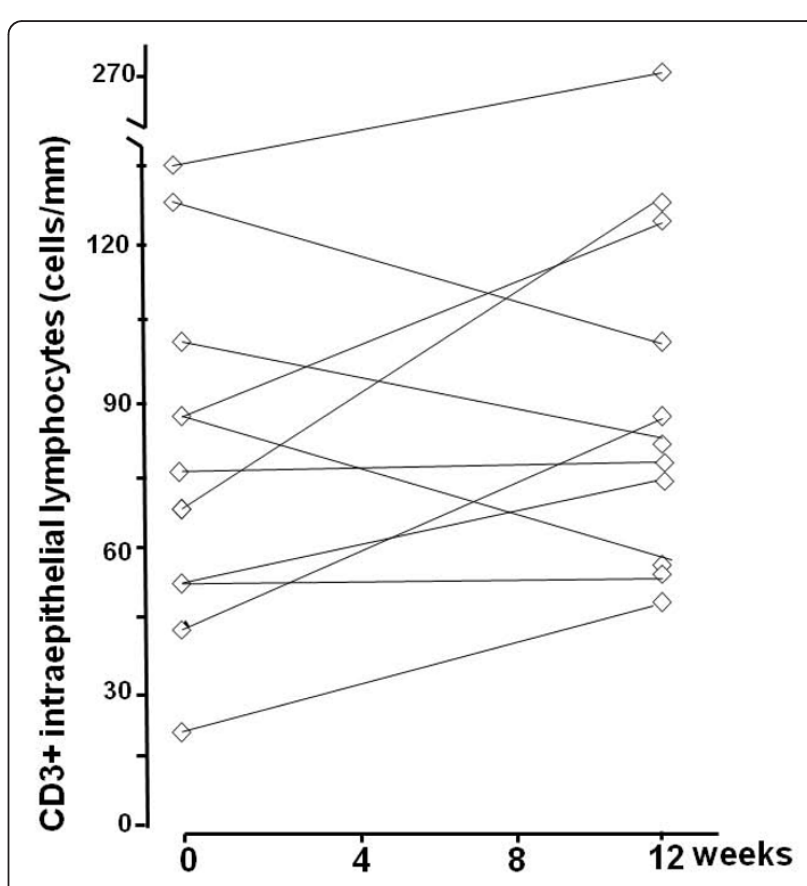

Figure 4 Changes in small bowel mucosal densities of CD3+ IELs in low-dose group at baseline and at completion of the study. 


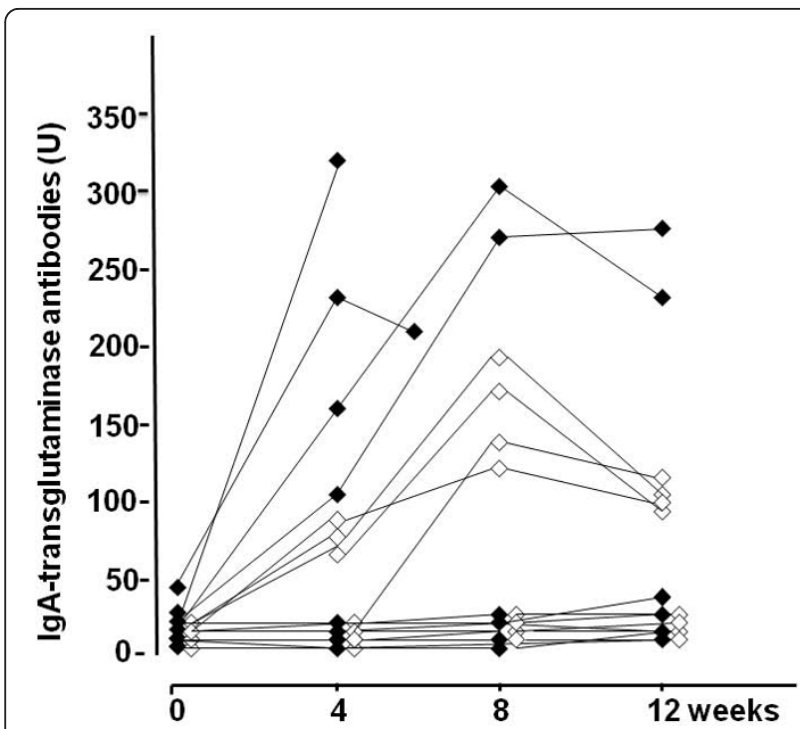

Figure 5 Changes in antibody levels. Transglutaminase 2 (TG2) antibody levels at baseline, at 4 and 8 weeks, and at the end of the study. Closed squares indicate moderate daily gluten dose 3-5 g and open squares low gluten doses (1-3 g per day).

three antibodies appeared in the serum simultaneously (data not shown). Small-bowel mucosal TG2-targeted autoantibody deposits generally followed the gluteninduced serological response in sera and morphological changes seen in the biopsy specimens (Tables 2 and 3).

At the outset, all subjects were free of gastrointestinal symptoms. Altogether 15 (71\%) out of the 21 celiac disease patients experienced mild to moderate abdominal symptoms upon gluten challenge; eight of them (including the four withdrawing prematurely, see above) were from the moderate-dose and seven from the low-dose group (Table 2). None developed severe symptoms. Thirteen (87\%) out of the 15 symptomatic patients also developed small-intestinal mucosal changes (significant decrease in $\mathrm{Vh} / \mathrm{CrD}$ and/or increase in the density of CD3+ IELs). Of note, symptoms did not reveal four celiac disease patients having clinically significant gluteninduced small-bowel mucosal damage.

\section{Discussion}

Twelve weeks' gluten challenge with both moderate and low amounts of gluten sufficed to induce small-bowel mucosal morphological damage in the majority of volunteer treated celiac disease adults not, however, in all. An increase in the density of CD3+ IELs paralleled this phenomenon especially in the moderate-dose gluten challenge group. At the same time, moderate gluten doses triggered more gastrointestinal symptoms leading to premature withdrawals from the study. This suggests that in the 12-week time-frame smaller gluten doses of 1-3 g per day might be preferable to higher doses in order to obtain sufficient complete read-outs at the end of a challenge study.

In this study, small-bowel mucosal $\mathrm{Vh} / \mathrm{CrD}$ measurement seemed to be the most sensitive parameter in both moderate and low gluten doses in assessing gluteninduced damage in celiac disease. This conception is also in line with previous findings, where the $\mathrm{Vh} / \mathrm{CrD}$ index has been used to show harmful effects of gluten in both short-term gluten challenges and long-term follow-up studies $[8,13,26,27]$. It is not surprising that the IEL count was a reliable marker for gluten toxicity only with moderate, but not with low gluten doses, as it has previously been shown that in celiac disease during a glutenchallenge, IELs accumulate in the surface and crypt epithelium in a dose-dependent manner [3]. It is also important to note that even if the majority of celiac disease patients after gluten challenge yielded positive readouts both in the Vh/CrD index and in the IEL count, there were some discrepant cases responding only with one or other parameter (Table 2). Marsh classification entailing six diagnostic categories (type 0-type 3c) has been widely used in the diagnostic work-up and followup of celiac disease [3]. Marsh grades type 1-type 3c all indicate cursorily that small-bowel mucosal IEL density is increased ( $>40$ IELs/100enterocytes), but different categories point more specifically to different morphological features (such as normal villous morphology, crypt hyperplasia or villous atrophy). Nonetheless the Marsh classification does not take into account minor gluteninduced mucosal changes (e.g. a significant decrease in $\mathrm{Vh} / \mathrm{CrD}$ or increase in density of IELs within one Marsh grade) or discrepant results in $\mathrm{Vh} / \mathrm{CrD}$ or IEL counts during a gluten challenge. It would thus be advisable in gluten challenge research projects, to use two separate continuous readout parameters in assessing glutendependent functional mucosal changes, i.e. the morphological $\mathrm{Vh} / \mathrm{CrD}$ and the inflammatory IEL changes, as opposed to applying a simplified grouped classification.

Even if abdominal symptoms after the consumption of cereals do not generally constitute a specific marker of untreated celiac disease [28], gluten-induced gastrointestinal symptoms in our challenge study showed high positive predictive value (87\%) for small-bowel mucosal injury in celiac disease patients. However, in $22 \%$ of the cases who developed significant small-intestinal damage, symptoms remained absent. It is widely known that many untreated celiac disease patients do not suffer from symptoms even if they have a manifest gluten-induced small-bowel mucosal lesion [29]. Furthermore, in some treated celiac disease patients, symptoms may disappear on a strict gluten-free diet despite continued ongoing mucosal villous atrophy $[30,31]$. Such persistent smallintestinal villous atrophy in symptom-free patients clearly carries a risk of subsequent severe complications such as 
osteoporosis and malignancies [30]. This warrants caution in relying solely on symptoms or other patientrelated outcomes without small-bowel biopsies in gluten challenge research projects.

In studies where a gluten challenge has been applied for diagnostic purposes in children on a normal daily glutencontaining diet (10-20 g daily), a small-bowel mucosal histological relapse has usually developed within three to twelve months in the majority of patients $[8,9]$. Seroconversion of EMA and TG2 antibodies is known to be a reliable predictor of histological relapse and antibody testing has helped in the timing of the biopsy [9]. In this study with low to moderate gluten doses, only $43 \%$ of the challenged adult celiac disease patients evinced a significant serological response. In line with earlier challenge studies, here all those who had increased serum autoantibodies during the challenge also developed small-bowel villous atrophy. However, at the same time serology missed nine cases with significant small-bowel mucosal changes (Tables 2 and 3). These cases might have called for a longer gluten challenge period or higher gluten amounts. However, it is well-known that serology may fail to detect occasional slight dietary transgressions and incomplete mucosal healing $[30,32,33]$, and therefore celiac serology is not always a reliable means of assessing gluten-induced harm. Furthermore, it is important to note that after the gluten challenge all three antibody - EMA, TG2 and DPG antibody - tests performed similar way, and recently introduced DPG antibody test was not superior to conventional serological assays. As small-bowel mucosal TG2-specific autoantibody deposits have also been found in seronegative celiac patients and patients with early-stage celiac disease $[23,24,34]$, it has been hypothesized that these mucosal autoantibodies might also appear early during a gluten challenge. In our study, mucosal autoantibodies followed the morphological damage, and they added only little to the normal morphometrical measurements. It might be that after a short-term acute gluten challenge the distribution of mucosal autoantibody depositions is patchy, and single mucosal biopsies may not reveal the positivity. It remains to be seen whether they will be of use at earlier time-points in the gluten challenge, but due to the invasive nature of small-bowel biopsies we were not able to take specimens on several occasions during the challenge.

There were some celiac disease patients who did not respond in terms of any of the measured outcome variables during the 12 weeks' gluten challenge. It is wellknown that celiac patients show varying sensitivity to gluten and the process of mucosal deterioration may take years or even decades [35-37]. Furthermore, it should be remembered that the phenotype of the disease may vary in the same person over time; celiac patients suffering originally from abdominal symptoms at diagnosis may later experience only extraintestinal symptoms such as dermatitis herpetiformis when gluten is re-introduced into the diet [37]. These issues need to be taken into account when sample sizes in study cohorts are determined.

At the baseline of the study, the majority of the treated celiac disease patients evinced increased densities of IELs and one patient even had villous atrophy with crypt hyperplasia despite adherent to a strict long-term gluten-free diet. According to the literature, such cases exist and it has been shown that even $80 \%$ of treated patients may have ongoing villous atrophy during a strict diet [38-40]. These persistent mucosal changes might be due to constant residual gluten contamination in the food, which again would indicate that alternative treatment options would be desirable.

\section{Conclusions}

All in all, further studies are still needed to establish the optimal gluten dose on the other hand causing some small-bowel mucosal deterioration but without inducing excessive ill health. Our results suggest that in drug trials, the duration of the gluten challenge might be shorter than 12 weeks and the gluten dose could be an average of those used in the current study (approximately $3 \mathrm{~g}$ ). As there are always some celiac disease patients who will not respond within these conditions, sample sizes must be sufficiently large to ensure adequate statistical power for the study. The Vh/CrD index seemed to be the most reliable outcome measurement in both moderate and low gluten doses. Furthermore, it must be remembered that during a gluten challenge, some celiac patients may remain asymptomatic even if they develop manifest gluten-induced mucosal damage - this might in the long-run predispose these cases to celiac disease-related complications.

\section{Abbreviations}

IELs: intraepithelial lymphocytes; Vh/CrD: villous height crypt depth ratio; TG2: transglutaminase 2; EMA: endomysium antibodies; DGP: deaminated gliadin peptide; ELISA: enzymelinked immunosorbent assay; Cl: confidence interval.

\section{Acknowledgements}

The Celiac Disease Study Group and this study are supported by the Academy of Finland Research Council for Health, the Competitive Research Funding of the Pirkanmaa Hospital District and the Sigrid Juselius Foundation.

\section{Author details}

'Paediatric Research Centre and School of Medicine, University of Tampere and Tampere University Hospital, Tampere, Finland. ${ }^{2}$ School of Health Sciences, University of Tampere, Finland. ${ }^{3}$ Department of Gastroenterology and Alimentary Tract Surgery, Tampere University Hospital and School of Medicine, University of Tampere, Tampere, Finland.

\section{Authors' contributions}

MLL participated in the study design, carried out the patient visits and helped to draft the manuscript. MM was involved in study design and preparing the manuscript. $\mathrm{KL}$ was involved in laboratory assessments and critically reviewed the manuscript. $\mathrm{HH}$ performed the statistical analysis and 
reviewed the manuscript critically. KK participated in the study design, was involved in the laboratory assessments and drafted the manuscript. All authors read and approved the final manuscript.

\section{Competing interests}

The authors declare that they have no competing interests.

Received: 10 August 2011 Accepted: 24 November 2011 Published: 24 November 2011

\section{References}

1. Rostom A, Murray JA, Kagnoff MF: The American Gastroenterological Association (AGA) Institute technical review on the diagnosis and management of celiac disease. Gastroenterology 2006, 131:1981-2002

2. Walker-Smith JA, Guandalini S, Schmitz J, Shmerling DH, Visakorpi JK: Revised criteria for diagnosis of coeliac disease. Arch Dis Child 1990, 65:909-11.

3. Marsh MN, Crowe PT: Morphology of the mucosal lesion in gluten sensitivity. Baillieres Clin Gastroenterol 1995, 9:273-93.

4. MacDonald WC, Brandborg LL, Flick AL, Trier JS, Rubin CE: Studies of celiac disease sprue. IV. The response of whole length of the small bowel to a gluten-free diet. Gastroenterology 1964, 47:573-89.

5. Murray JA, Rubio-Tapia A, Van Dyke $C T$, et al: Mucosal atrophy in celiac disease: extent of involvement, correlation with clinical presentation, and response to treatment. Clin Gastroenterol Hepatol 2008, 6:186-93.

6. Farrell RJ, Kelly CP: Celiac sprue. N Engl J Med 2002, 346:180-8.

7. McNeish AS, Harms HK, Rey J, Shmerling DH, Visakorpi JK, Walker-Smith JA: The diagnosis of coeliac disease. A commentary on the current practices of members of the European Society for Paediatric Gastroenterology and Nutrition (ESPGAN). Arch Dis Child 1979, 54:783-6.

8. Holm K, Mäki M, Vuolteenaho $\mathrm{N}$, et al: Oats in the treatment of chilhood coeliac disease: a two-year controlled and a long-term clinical follow-up study. Aliment Pharmacol Ther 2006, 23:1463-72.

9. Mäki M, Lähdeaho ML, Hällström O, Viander M, Visakorpi JK: Postpubertal gluten challenge in coeliac disease. Arch Dis Child 1989, 64:1604-7.

10. van Overbeek FM, Uil-Dieterman IG, Mol IW, Kohler-Brands L, Heymans HS, Mulder CJ: The daily gluten intake in relatives of patients with coeliac disease compared with that of the general Dutch population. Eur $J$ Gastroenterol Hepatol 1997, 9:1097-9.

11. Kumar PJ, Walker-Smith J, Milla P, Harris G, Colyer J, Halliday R: The teenage coeliac: follow up study of 102 patients. Arch Dis Child 1988, 63:916-20.

12. Montgomery AM, Goka AK, Kumar PJ, Farthing MJ, Clark ML: Low gluten diet in the treatment of adult coeliac disease: effect on jejunal morphology and serum anti-gluten antibodies. Gut 1988, 29:1564-8.

13. Catassi C, Fabiani E, lacono G, et al: A prospective, double-blind, placebocontrolled trial to establish a safe gluten threshold for patients with celiac disease. Am J Clin Nutr 2007, 85:160-6.

14. Hall NJ, Rubin G, Charnock A: Systematic review: adherence to a glutenfree diet in adult patients with coeliac disease. Aliment Pharmacol Ther 2009, 30:315-30.

15. Collin P, Thorell L, Kaukinen K, Mäki M: The safe threshold for gluten contamination in gluten-free products. Can trace amounts be accepted in the treatment of coeliac disease? Aliment Pharmacol Ther 2004, 19:1277-83.

16. Hischenhuber C, Crevel R, Jarry B, et al: Review article: safe amounts of gluten for patients with wheat allergy or coeliac disease. Aliment Pharmacol Ther 2006, 23:559-75.

17. Sollid LM, Khosla C: Novel therapies for coeliac disease. J Intern Med 2011, 269:604-13.

18. Aziz I, Evans KE, Papageorgiou V, Sanders DS: Are patients with coeliac disease seeking alternative therapies to a gluten-free diet? $J$ Gastrointestin Liver Dis 2011, 20:27-31.

19. Kuitunen P, Kosnai I, Savilahti E: Morphometric study of the jejunal mucosa in various childhood enteropathies with special reference to intraepithelial lymphocytes. J Pediatr Gastroenterol Nutr 1982, 1:525-31.

20. Kaukinen $\mathrm{K}$, Peräaho $\mathrm{M}$, Collin $\mathrm{P}$, et al: Small bowel mucosal transglutaminase 2-specific lgA deposits in coeliac disease without villous atrophy: a prospective and randomized study. Scand $J$ Gastroenterol 2005, 40:564-572.

21. Järvinen $\Pi$, Kaukinen $\mathrm{K}$, Laurila $\mathrm{K}$, et al: Intraepithelial lymphocytes in celiac disease. Am J Gastroenterol 2003, 98:1332-7.
22. Sulkanen $\mathrm{S}$, Halttunen $\mathrm{T}$, Laurila $\mathrm{K}$, et al: Tissue transglutaminase autoantibody enzyme-linked immunosorbent assay in detecting celiac disease. Gastroenterology 1998, 115:1322-8.

23. Korponay-Szabo IR, Halttunen T, Szalai Z, et al: In vivo targeting of intestinal and extraintestinal transglutaminase 2 by coeliac autoantibodies. Gut 2004, 53:641-8.

24. Salmi TT, Collin P, Korponay-Szabo I, et al: Endomysial antibody-negative coeliac disease: clinical characteristics and intestinal autoantibody deposits. Gut 2006, 55:1746-53.

25. Shiner $M$, Ballard J: Antigen-antibody reactions in jejunal mucosa in childhood coeliac disease after gluten challenge. Lancet 1972, 1:1202-5.

26. Kaukinen K, Collin P, Holm K, et al: Wheat starch-containing gluten-free flour products in the treatment of coeliac disease and dermatitis herpetiformis. A long-term follow-up study. Scand J Gastroenterol 1999, 34:164-169.

27. Sturgess $\mathrm{R}$, Day $\mathrm{P}$, Ellis $\mathrm{HJ}$, et al: Wheat peptide challenge in coeliac disease. Lancet 1994, 343:758-61.

28. Kaukinen $\mathrm{K}$, Turjanmaa $\mathrm{K}$, Mäki M, et al: Intolerance to cereals is not specific for coeliac disease. Scand I Gastroenterol 2000, 35:942-6.

29. Mäki M, Mustalahti K, Kokkonen J, et al: Prevalence of celiac disease among children in Finland. N Engl J Med 2003, 348:2517-24.

30. Kaukinen $\mathrm{K}$, Peräaho $\mathrm{M}$, Lindfors $\mathrm{K}$, et al: Persistent small bowel mucosal villous atrophy without symptoms in coeliac disease. Aliment Pharmacol Ther 2007, 25:1237-45.

31. Rubio-Tapia A, Rahim MW, See JA, Lahr BD, Wu TT, Murray JA: Mucosal recovery and mortality in adults with celiac disease after treatment with a gluten-free diet. Am J Gastroenterol 2010, 105:1412-20.

32. Dickey W, Hughes DF, McMillan SA: Disappearance of endomysial antibodies in treated celiac disease does not indicate histological recovery. Am J Gastroenterol 2000, 95:712-4.

33. Troncone R, Mayer M, Spagnuolo F, Maiuri L, Greco L: Endomysial antibodies as unreliable markers for slight dietary transgressions in adolescents with celiac disease. J Pediatr Gastroenterol Nutr 1995, 21:69-72.

34. Salmi TT, Collin $\mathrm{P}$, Järvinen $\mathrm{O}$, et al: Immunoglobin $\mathrm{A}$ autoantibodies against transglutaminase 2 in the small intestinal mucosa predict forthcoming coeliac disease. Aliment Pharmacol Ther 2006, 24:541-52

35. Högberg L, Stenhammar L, Wagermark J: Very late mucosal relapse in a girl with coeliac disease. Acta Paediatr 1993, 82:887-9.

36. Matysiak-Budnik T, Malamut G, Patey-Mariaud de Serre N, et al: Long-term follow-up of 61 patients diagnosed in childhood:evolution towards latency is possible on a normal diet. Gut 2007, 56:1379-86.

37. Kurppa $\mathrm{K}$, Koskinen $\mathrm{O}$, Collin $\mathrm{P}$, et al: Changing phenotype of celiac disease after long-term gluten exposure. J Pediatr Gastroenterol Nutr 2008, 47:500-3.

38. Bardella MT, Velio P, Cesana BM, et al: Coeliac disease: a histological follow-up study. Histopathology 2007, 50:465-71.

39. Lee SK, Lo W, Memeo L, Rotterdam H, Green PHR: Duodenal histology in patients with celiac disease after treatment with a gluten-free diet. Gastrointest Endosc 2003, 57:187-91.

40. Lanzini A, Lanzarotto F, Villanacci V, et al: Complete recovery of intestinal mucosa occurs very rarely in adult coeliac patients despite adherence to gluten-free diet. Aliment Pharmacol Ther 2009, 29:1299-308.

\section{Pre-publication history}

The pre-publication history for this paper can be accessed here: http://www.biomedcentral.com/1471-230X/11/129/prepub

\section{doi:10.1186/1471-230X-11-129}

Cite this article as: Lähdeaho et al: Small- bowel mucosal changes and antibody responses after low- and moderate-dose gluten challenge in celiac disease. BMC Gastroenterology 2011 11:129. 